\title{
Kernos
}

Revue internationale et pluridisciplinaire de religion grecque antique

6 | 1993

Varia

\section{Aubriot-Sévin, Prière et conception religieuse en Grèce ancienne}

\section{Paul Wathelet}

\section{(2) OpenEdition \\ Journals}

\section{Édition électronique}

URL : http://journals.openedition.org/kernos/565

DOI : 10.4000/kernos.565

ISSN : 2034-7871

\section{Éditeur}

Centre international d'étude de la religion grecque antique

\section{Édition imprimée}

Date de publication : 1 janvier 1993

Pagination : 379-381

ISSN : 0776-3824

\section{Référence électronique}

Paul Wathelet, «D. Aubriot-Sévin, Prière et conception religieuse en Grèce ancienne », Kernos [En ligne], 6 | 1993, mis en ligne le 07 avril 2011, consulté le 24 septembre 2020. URL : http:// journals.openedition.org/kernos/565 ; DOI : https://doi.org/10.4000/kernos.565 


\section{Notices bibliographiques}

Danièle AUBRIOT-SÉVIN, Prière et conceptions religieuses en Grèce ancienne jusqu'à la fin du Ve siècle av. J.-C., Lyon-Paris, Maison de l'Orient Méditerranéen-de Boccard, 1992, 1 vol. 17 × $25 \mathrm{~cm}, 604$ p. (Collection de la Maison de l'Orient Méditerranéen, 22. Série littéraire et philosophique, 5). ISBN : 2-903264-14-7.

Les spécialistes attendaient avec impatience la publication du doctorat d'État de Danièle Aubriot; ils ne seront pas déçus. Il s'agit d'une somme qui reprend tout le problème de la prière et de ses implications en Grèce jusqu'à la fin du Ve siècle. Après une introduction qui fait le point des nombreuses études sur le sujet, l'ouvrage comprend cinq chapitres. Le premier étudie les circonstances de la prière (prières cultuelles et prières libres; personne et condition des orants; circonstances matérielles; lieu, moment, préparatifs; occasions qui déterminent une prière, objet et contenu de la requête). Le deuxième porte sur l'expression corporelle et l'expression vocale de la prière (gestes et attitudes, larmes; le timbre et le ton de la voix; hymne, cri et prière). Avec le troisième chapitre, l'A. aborde des questions formelles, elle s'attache au sens de $\varepsilon$ v̌xo $\mu \alpha \imath$ (prière et discours, la formulation de la requête). Le quatrième chapitre est consacré à $\alpha \alpha^{\prime} \alpha{ }_{0} \mu \alpha$ et à $\alpha \rho \alpha ́$, à la malédiction et au serment (les emplois de $\alpha \rho \alpha ́ \alpha \mu \alpha \iota$ dans les textes, chez Homère, Pindare, Sappho et Hérodote; nature et fonctionnement de l'ó $\rho \dot{\alpha}$ et du serment). Le cinquième chapitre examine la supplication et les démarches conciliaires ou piaculaires (la supplication et ses différents rites, la supplication du meurtrier, le port des rameaux, la position assise, la supplication aux morts; $\lambda i \sigma \sigma o \mu \alpha \imath$ et ses différents emplois selon les textes; $\lambda i ́ \sigma \sigma o \mu \alpha \imath$ et

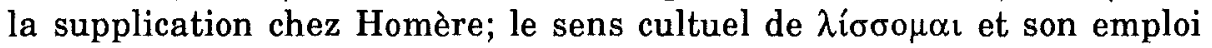

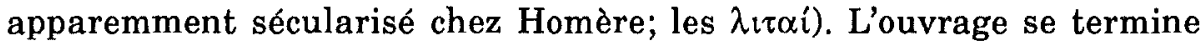
par des conclusions substantielles. Il comporte, en appendice, un corpus des occurrences de ápóo $\mu \alpha \imath$ dans l'épopée, chez Sappho, Hérodote, Eschyle, Sophocle, Euripide et Aristophane, des index des nombreux passages d'auteurs cités ou commentés et de mots, noms et expressions ou notions utiles.

Le simple énoncé de la table des matières du livre en dit toute la richesse et la profondeur. L'A., qui fait preuve d'une connaissance exceptionnelle de la littérature grecque, s'appuie sur une exégèse des textes pleine de finesse et qui resitue les passages dans l'ensemble des œuvres. Elle fait une analyse critique, minutieuse et justifiée, mais parfois un peu longue, de toutes les études antérieures, et l'ampleur du corps des notes au bas des pages, de même que l'importance de la biblio- 
graphie citée en fin de volume, témoignent de la richesse de sa documentation.

La réunion de toutes ces données permet à l'A. de réfuter, ou au moins de nuancer, des affirmations tranchées qui, à force d'être répétées de manuel en manuel ont pris valeur de vérités. C'est spécialement le cas dans les deux premiers chapitres où l'examen des documents constitue un rappel salutaire à la prudence. L'A. note, entre autres, l'absence de formalisme qui caractérise la prière (tout le monde peut prier, à condition d'éviter l'v̌ $\beta \rho ı s)$, elle souligne la valeur du discours articulé. Mais la même prudence est de mise dans la question de savoir si la prière était formulée à haute voix ou non. L'idée répandue que les gestes et les attitudes sont commandés par la nature des dieux invoqués ne résiste pas à l'examen, ils dépendent plutôt de la nature même de la prière, dont il convient de souligner la variété et la richesse, et qui peut se combiner avec la danse et le chant. Le schéma tripartite de la prière, qui apparaît notamment dans l'épopée, n'a rien de liturgique, contrairement à ce qui est souvent affirmé.

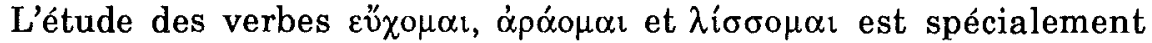

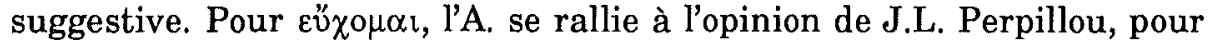
lequel le verbe a une origine sociale. Le sujet exprime une revendication jugée légitime. Le verbe est ensuite passé dans le domaine religieux, ce qui implique le caractère anthropomorphique des dieux

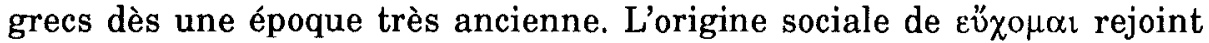
la gestuelle persuasive de certaines prières. L'analyse de $\alpha$ póopoı est féconde. L'A. suggère de traduire d́ó́o $\mu \alpha \iota$, non par "prier", mais bien par "proférer un souhait solennel", "souhaiter solennellement», ce qui explique que l'ópó n'intervienne pas dans le rituel du sacrifice; elle est la parole douée d'une valeur curative à portée sociale, qui vient en aide à la $\Delta i ́ k \eta$. Ainsi s'explique qu'en étant punitive, elle devienne malédiction. En manifestant sans doute une réserve excessive à l'égard des thèses de Milman Parry et sans analyser en détail l'emploi formulaire du verbe, l'A. remarque qu'Homère a fait dériver le sens d'ópóo $\mu \alpha \iota$ pour lui conférer la valeur d'une demande d'assistance immédiate à la divinité. Quand on sait les réticences qu'Homère manifeste vis-à-vis des formes participatives de la religion, une telle opinion surprend. En ce qui concerne $\lambda i ́ \sigma \sigma o \mu \alpha \imath$ l'A. rejette à la fois la thèse selon laquelle le verbe désignerait des rapports "entre pairs" et celle qui lui donne le sens de "supplier", en en faisant un synonyme de ívéopa. Alors que la supplication est contraignante, $\lambda i \sigma^{\sigma} \sigma o \mu \alpha_{\imath}$ implique une demande en grâce, non contraignante; les deux valeurs peuvent se rejoindre, mais elles sont fondamentalement différentes. Contrairement à ce que ferait croire l'usage homérique, $\lambda i \sigma_{\sigma o} \mu \alpha \imath$ a d'abord une valeur religieuse, ce

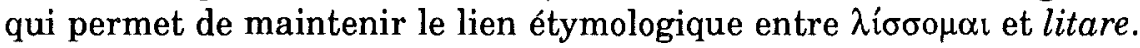


L'A. insiste avec raison sur l'importance de l'épopée homérique qui a fortement humanisé et personnalisé les dieux. L'œuvre d'Homère a marqué profondément le reste de la littérature grecque et le poète exprime, en cette matière comme en d'autres, des positions originales qui ne correspondent pas à ce que ferait attendre son époque.

En reconnaissant les différences essentielles qui existent entre nos conceptions religieuses et celles des Grecs, l'A. souligne enfin le souci de la religion grecque de «maintenir, à travers cette communication même [la prière], une distance que rien ne doit compromettre", et le rôle

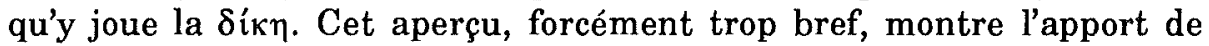
l'ouvrage qu'aucun spécialiste de la religion grecque ne pourra ignorer.

Paul WATHELET (Liège)

\section{Aphrodite AVAGLANOU, Sacred Marriage in the Rituals of Greek Religion, Bern, Peter Lang, 1991, 1 vol. 15,3 x 22,5 cm, XV+260 p., 9 dessins (European University Studies. Series 15. Classics, 54). ISBN : 3-261-04432-2.}

Cette dissertation doctorale, dirigée par W. Burkert, se propose de

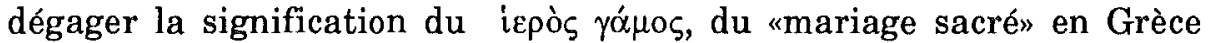
ancienne. En effet, depuis Mannhardt, le sens de cette expression aurait été beaucoup trop étendu, avec Frazer et Klinz notamment, allant jusqu'à désigner tout type d'union en relation avec un contexte magique de fertilité et dont un des partenaires est de nature divine. Pour l'A., le caractère sacré du yó $\mu_{0}$ implique que les deux partenaires doivent être divins - ce qui exclut toute une série de vagabondages divins en territoire humain. De plus, la notion de $\gamma \alpha \dot{\alpha} \mu о \varsigma$ en tant que mariage institutionnalisé et durable exclut les relations fondées sur un rapt. Il s'agit donc de chercher à quels cas précis ces deux principes peuvent être appliqués et quelles différences existent entre le "mariage sacré» et d'autres types de $\mu i \xi_{\varepsilon} \iota \zeta$. Enfin, contre l'interprétation par la «magie sympathique", l'A. entend souligner le caractère initiatique de ce type de mariage.

Puisqu'il s'agit d'étudier un $\gamma \alpha \dot{\alpha} \mu \rho \varsigma$, l'introduction tente, dans une première partie, de reconstituer le plus complètement possible la cérémonie du mariage qui, pour les humains, est une affaire légale, sociale, mais dont le contenu religieux est loin d'être négligeable. Sur base de sources littéraires et de la peinture de vases, la présentation

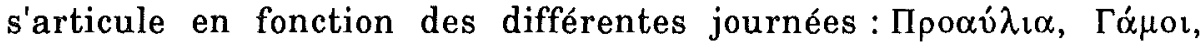

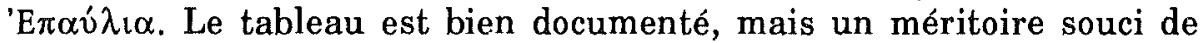
clarté s'affirme parfois au mépris de la complexité des problèmes : les sacrifices préliminaires ne sont pas une rétribution pour la perte de la chasteté (p. 4) (le mariage se doit d'être chaste !), mais bien celle de la virginité; il n'est pas sûr que le loutrophore placé sur la tombe des 